\title{
The Role of the Right Anterior Prefrontal Cortex in Episodic Retrieval
}

\author{
K. Allan,* R. J . Dolan,† P. C. Fletcher,† and M. D. Ruggł \\ *Department of Psychol ogy, University of Aberdeen, Aberdeen AB24 2UB; †Wel lcome Department of Cognitive Neurol ogy, London; \\ and $\ddagger$ Institute of Cognitive Neuroscience and Department of Psychology, University Coll ege London, London, United Kingdom
}

Received J une 18, 1999

\begin{abstract}
Regional brain activity was measured with $\mathrm{H}_{2}{ }^{15} \mathrm{O}$ PET while participants attempted to complete wordstem and word-fragment retrieval cues with previously studied words. The retrieval cue manipulation was employed to gain control over the monitoring operations associated with evaluating the episodic status of alternative cue completions. These operations were more constrained for fragments, which had fewer possible completions than each corresponding stem. In one condition (zero target), during the scanning interval none of the cues could be completed with studied items, whereas in another condition (high target), $80 \%$ of cues belonged to studied items. Relative to baseline tasks, right anterior prefrontal activity was greater for stems than for fragments in the zero target condition. The target density manipulation did not modulate right anterior prefrontal activity, but was associated with increased activity in right dorsolateral prefrontal cortex. These findings are consistent with the proposal that the right anterior prefrontal cortex supports monitoring operations during episodic retrieval tasks. In addition, the findings add to evidence suggesting that the dorsolateral and anterior right prefrontal cortex make functionally distinct contributions to episodic retrieval. 2000 Academic Press
\end{abstract}

\section{INTRODUCTION}

It has consistently been reported in functional neuroimaging studies of memory that episodic retrieval is associated with activation of right prefrontal cortex (see for reviews Buckner and Koutstall, 1998; Fletcher et al., 1997). The regions most commonly involved in this effect include anterior lateral cortex [Brodmann area (BA) 10) and dorsolateral cortex of the middle frontal gyrus (BA 46/9)]. The functional significance of these findings has been the subject of debate. They have for example been interpreted as reflecting the adoption of a "retrieval mode" (Nyberg et al., 1995), the expenditure of "retrieval effort" (Schacter et al., 1996), and the engagement of "postretrieval" processing (Rugg et al., 1996).
Rugg et al. (1998) proposed a modification to the postretrieval hypothesis to account for the patterns of activation observed in right anterior prefrontal cortex (PFC) during different episodic retrieval tasks. According to the "monitoring" hypothesis, this region supports cognitive operations that evaluate whether retrieved information belongs to a prior "target" episode (see Henson et al., 1999a; Fletcher et al., 1995a; for similar proposals with respect to right dorsolateral prefrontal cortex).

The monitoring hypothesis was suggested by the task-specific patterns of right anterior PFC activity observed by Rugg et al. (1998) in a PET study of cued recall and recognition memory. Both tasks had two memory conditions and one baseline condition. For cued recall, in both of the memory conditions wordstem (e.g., MOT_) retrieval cues were completed, if possible, with previously encoded words (e.g., MOTOR). In the high target memory condition, during the scanning period approximately $80 \%$ of the stems belonged to studied items, while in the zero target memory condition none of the stems presented during this period belonged to studied items. The baseline condition required stems belonging to unstudied words to be completed with the first suitable word to come to mind. F or recognition memory, studied and unstudied words had to be judged as "old" or "new," respectively. As for cued recall, the ratio of studied to unstudied items was manipulated during the scanning period to form a high and a zero target condition. The recognition baseline condition required unstudied words to be read al oud.

For stem cued recall, right anterior PFC was more active in the zero target condition relative to the high target and the baseline conditions. Rugg et al . proposed that this finding reflected the adoption of a "generate/ recognize" strategy, whereby the episodic status of alternative completions for each stem (e.g., MOTEL, MOTIVATE, etc., for MOT_) was iteratively evaluated until either the correct completion was found or the search was terminated. The monitoring of retrieval products during attempts to recall, and associated right anterior PFC activity, would thus have been maximal in the zero target condition because none of the re 
trieval cues presented during the scanning interval belonged to target items. During the high target condition, monitoring would have been limited by the successful retrieval of target items on approximately $50 \%$ of trials. And in the baseline condition, there was no need to evaluate the episodic status of each completion. During the recognition task, the generation of multiple candidate target items was precluded because wholeword "copy" cues were provided, and thus postretrieval monitoring operations would have been confined to the high target condition, in which the level of accurate recognition was approximately $90 \%$. Accordingly, Rugg et al. found that during the recognition task, the right anterior PFC was more active in the high target condition relative to both the zero target and the baseline conditions, which did not differ from one another in this respect. Rugg et al . (1996) also observed increased right anterior PFC activity during recognition memory when contrasting high and low target conditions.

The present experiment employs PET and the same general design as in the study of Rugg et al. (1998), to explore further the link between retrieval monitoring and right anterior PFC activity. We employed a retrieval cue manipulation that was designed to affect postretrieval monitoring during two versions of a cued recall task. For each target word to be recalled, a word stem and a "word fragment" (e.g., M-TO- for MOTOR) retrieval cue were created, such that each fragment had many fewer possible completions than each stem. Thus, when attempting to recall target items using the stems as retrieval cues, participants will be able iteratively to evaluate more candidate items than when using the fragments. Therefore, when the retrieval of a target item is not possible, as in the zero target conditions, increased right anterior PFC activity is predicted during stem cued recall compared to fragment cued recall, relative to their respective baseline conditions.

Monitoring operations may also be differentially engaged in the high target conditions of stem and fragment cued recall, relative to their respective zero target conditions. As argued above, during stem cued recall such operations will predominate in the zero relative to the high target condition. But for fragment cued recall, postretrieval monitoring should beconfined mainly to the high target condition. This is because the level of monitoring will vary with attempts to retrieve, irrespective of whether the retrieved information is judged as bel onging to a target episode. Hence, the lack of available alternative completions for the fragments will act to constrain retrieval attempts per se. Thus, there should be only appreciable retrieval products to monitor in the high target fragment condition in which approximately $80 \%$ of cues belong to study items and then only to the extent that retrieval is successful. An additional prediction of the monitoring hypothesis is therefore that increased activity in the right anterior PFC should be observed in the high target relative to the zero target condition in fragment cued recall but not in stem cued recall.

\section{METHOD}

\section{General Design}

Each cued recall task had two memory conditions and one control condition. In the two memory conditions, the retrieval cues had to be completed whenever possible with items that had been presented during a prior encoding phase. In one of the memory conditions, in the middle portion of the test list approximately $80 \%$ of the cues belonged to studied items (high target). In the other memory condition, the middle portion of the test list was composed of cues that belonged exclusively to unstudied items (zero target). The beginning and final portions of each test list comprised a 50:50 mixture of cues belonging to studied and unstudied items. The baseline conditions required the retrieval cues to be completed with any suitable word to come to mind, matching the perceptual and the response requirements to those of the memory conditions.

\section{Participants}

Eight healthy young adult males took part in the experiment (all right handed, mean age 28.5 years, range 22-46). Written consent was given by each participant prior to the experimental session, and the study was approved by the local hospital ethics committee and the UK Administration of Radioactive Substances Advisory Committee.

\section{Stimuli}

The stimuli were taken from the pool of items employed by Rugg et al. (1998). Each item had a unique three-letter stem that could be used to form at least five different English words including the target item. A word fragment was created for each critical item by randomly replacing letters in each word with a hyphen character, al ways retaining the first letter and ensuring that no more than two consecutive letters remained in the resulting fragment. The number of letters replaced was equal to the integer value of the length of each item divided by 2 . The resulting fragments were then each checked to ensure that either they were uniquely completed by the target item or the number of alternative completions was less than the number of completions for the corresponding stem (e.g., MOT and M-TO- for the target item MOTOR).

Twelve unique sets of study and test lists, containing 21 and 34 items, respectively, were formed. The first 14 and last 6 items in each test list comprised equal 
numbers of cues belonging to studied and unstudied items, in a different randomized order in each list. Two pairs of test lists corresponded to each study list, one pair containing stems and the other containing fragments. One list from each pair was employed for the zero target condition and the other list was employed for the high target condition. In the zerotarget lists, the middle 14 cues bel onged exclusively to unstudied items, while in the high target lists 11 of the middle 14 items belonged to studied items. All stimuli were presented as black text on a white background on a TV monitor suspended approximately $45 \mathrm{~cm}$ in front of the subject. The study phase presentation rate was controlled by the experimenter, each item remaining on screen until it was replaced by the next one. The stimulus onset asynchrony during test was $3.5 \mathrm{~s}$ with a stimulus duration of $2 \mathrm{~s}$.

\section{Tasks}

During the control tasks, participants viewed a series of stems or fragments belonging to unstudied words and were asked to complete each with any suitable word. If a word could not be generated, then a don't know response was required. A study phase preceded each memory task. Each of the 21 items presented during the study phase was incorporated into a (novel) sentence, which was spoken aloud. The experimenter initiated the next trial once the sentence had been given. The memory task instructions required each retrieval cue to be completed with an item from the immediately preceding study phase. If this could not be done, the cue could be completed with any suitable item, and failing that a don't know response was required. Each completion had to be spoken aloud, followed by either old or new, to indicate whether the completion was judged to have been on the study list. The response requirements for each condition and type of memory cue were thus identical.

Each of the control and memory tasks was administered twice, giving a total of 12 study-test blocks, presented in a different ABCDEFFEDCBA order for each participant. A different study-test set was employed for each block, with the allocation of sets to blocks arranged such that each was presented equally often across the different control and memory conditions.

\section{Procedure}

Instructions for the memory tasks were given once the participant had been placed in the scanner and cannulated and an attenuation scan had been performed. A practice study (10 items) and test (10 items, 5 stems and 5 fragments belonging to the 10 practice study items) were then given to familiarize the participant with the tasks. Participants were not told that the proportion of cues belonging to studied items would be manipulated across blocks. The first experimental block was then given. Each study-test block lasted 8 min on average. The interval between finishing a block and beginning the next was used as a rest period. The beginning of the middle portion of each test list was timed to coincide with the beginning of the scanning period (see bel ow). The study/test interval was approximately $2 \mathrm{~min}$ on average. During this period each partici pant performed a distractor task invol ving counting backward in threes from an arbitrary number.

\section{PET Scanning}

PET scans were obtained with a Siemens/CPS ECAT EXACT HR + (Model 962) PET camera in 3D acquisition mode. In each scan, participants received a $20-5$ intravenous bolus of $\mathrm{H}_{2}{ }^{15} \mathrm{O}$ through a forearm cannula at a concentration of $55 \mathrm{MBq} \mathrm{ml}^{-1}$ and a flow rate of 10 $\mathrm{ml} \mathrm{min}^{-1}$. The infusion of the radioactive bolus and the presentation of each test were timed so that rCBF measurement began during the middle portion of each test list. Each scan produced an image of rCBF that was integrated over a 90-s period beginning from the time when the tracer first entered the cerebral circulation. The middle portion of each test list was presented during the initial $30 \mathrm{~s}$ of this period, when tracer uptake within the head rises. It is during this period that the distribution of the radioactive tracer within the brain is affected according to experimental condition (Silbersweig et al., 1993).

\section{PET Data Analyses}

The attenuation-corrected PET scans were realigned to correct for movement during the scanning period, then transformed into standard Tailarach and Tournoux (1988) space. The data were then smoothed with an isotropic Gaussian kernel of full-width half-maximum equal to $10 \mathrm{~mm}$. Statistical analyses of the PET data were performed using the general linear model as implemented in SPM97D (Wellcome Department of Cognitive Neurology, London, UK). Condition, subject, and covariate effects were estimated for each voxel, with differences in global CBF within and between subjects removed by analysis of covariance. The specific contrasts used to analyze condition effects are described under Results. Unless indicated otherwise, the one-tailed significance level adopted for all contrasts was $\mathrm{P}<0.001$ (uncorrected for multiple comparisons).

\section{RESULTS}

\section{Behavioral Data}

The performance data are given in Table 1 . The "baseline" rate provides an estimate of the chance probability of completing a stem with a target item, as measured by the proportion of stems belonging to 
TABLE 1

Behavioral Data

\begin{tabular}{|c|c|c|c|c|c|c|}
\hline & \multicolumn{3}{|c|}{ Stems } & \multicolumn{3}{|c|}{ Fragments } \\
\hline & Baseline & High & Zero & Baseline & High & Zero \\
\hline Recall & $9.9(4.2)$ & 57.4 (13.4) & 48.1 (11.0) & $13.8(5.8)$ & $54.2(7.6)$ & 49.3 (15.7) \\
\hline False alarm & - & $1.6(1.7)$ & $3.7(2.4)$ & - & $2.8(2.4)$ & $3.7(4.6)$ \\
\hline Don't know & $3.1(5.4)$ & $7.35(3.2)$ & $7.9(6.5)$ & 50.9 (16.3) & 38.6 (8.6) & 49.4 (14.8) \\
\hline
\end{tabular}

Note. Baseline, recall, falsealarm, and don't know rates (\%), plus SDs in parentheses, for stem and fragment cued recall in the high and zero target density conditions.

unstudied items that were completed with words bel onging to the experimental pool. The baseline rate was higher for fragments relative to stems, but this difference was not statistically significant $[F(1,7)=1.60$, $\mathrm{P}>0.2]$. The participants failed to provide a completion for fragments far more often than was the case for the stems, as indicated by the substantially higher don't know rates for fragments relative to stems shown in Table 1. This was confirmed by ANOVA of the don't know rates according to task and condition, which revealed a significant main effect of task [F(1, $7)=127.12, \mathrm{P}<0.001$ ] due to the greater rate for fragments. The ANOVA also revealed a significant interaction between the factors of task and condition $[F(1.9,13.4)=9.09, P<0.005]$ (degrees of freedom corrected for violations of sphericity by the GeisserGreenhouse method; Keselman and Rogan, 1980). Post hoc tests (Newman-Keuls method, $\mathrm{P}<0.05$ significance level) revealed that there were no significant differences in the don't know rates to stems between the baseline and the memory conditions. For fragments, however, the don't know rate in the high target condition was significantly lower than in the zero target and the baseline conditions, which did not differ from one another.

Table 1 also shows the recall and false alarm rates in each condition. The recall rate reflects the proportion of completions with studied items that attracted a correct old judgment (the proportion of correct completions that were not recognized was negligible and did not differ according to task or target density). The false alarm rate reflects the proportion of completions with unstudied items given an incorrect old judgment, summed across cues belonging to studied and unstudied items. The false alarm rate was subtracted from the recall rate as a guessing correction, and the corrected recall rates were then analyzed by ANOVA to determine whether the factors of task and target density had an effect upon memory performance. The only significant effect observed was a main effect of target density $[F(1,7)=10.55, P<0.025]$, due to the enhanced recall of studied items in the high relative to the zero density conditions for both tasks.

\section{PET Data}

Following the approach of Rugg et al. (1998), task engagement effects were first identified by pairwise contrasts between the zero target density and the baseline conditions (Table 2). Both tasks engaged the right anterior PFC with peak activations in closely

TABLE 2

X, y, and z Coordinates (Talairach and Tournoux, 1988), Z Values, Brain Regions, and Approximate Brodmann Areas (BAs) of the Peak I ncreases in rCBF for the Pairwise Contrasts between the Zero Target and the Baseline Conditions for Each Task

\begin{tabular}{clcllr}
\hline Contrast & Coordinates & Voxels & Z & \multicolumn{1}{c}{ Region } & BA \\
\hline Stem cued recall & $18,51,-15$ & 165 & 4.33 & Right anterior prefrontal & Left anterior prefrontal \\
& $-36,48,9$ & 5 & 3.39 & Right middle frontal gyrus & 10 \\
& $36,6,51$ & 9 & 3.69 & Right middle frontal gyrus & 8 \\
& $36,33,33$ & 9.63 & 4.90 & Medial parietal & 7 \\
& $0,-72,48$ & 71 & 3.78 & Posterior cingulate & 23 \\
& $0,-18,30$ & 18 & 4.20 & Left cerebellum & Right cerebellum \\
Fragment cued recall & $-9,-72,-24$ & 48 & 3.76 & Right anterior prefrontal & 10 \\
& $48,-51,-48$ & 12 & 3.84 & Right superior frontal gyrus & 9 \\
& $18,54,-6$ & 7 & 3.31 & Left posterior cingulate & 23 \\
& $22,42,30$ & 8 & 3.88 & Left middle temporal gyrus & 21 \\
\hline
\end{tabular}


adjacent regions of $\mathrm{BA} 10$ and within the right dorsolateral PFC in BA 8/9 (see Fig. 1).

Two additional contrasts were employed to reveal whether the engagement response within the right PFC differed according to task. These contrasts involved computing the interaction between the engagement effects (zero target - baseline) for stems versus
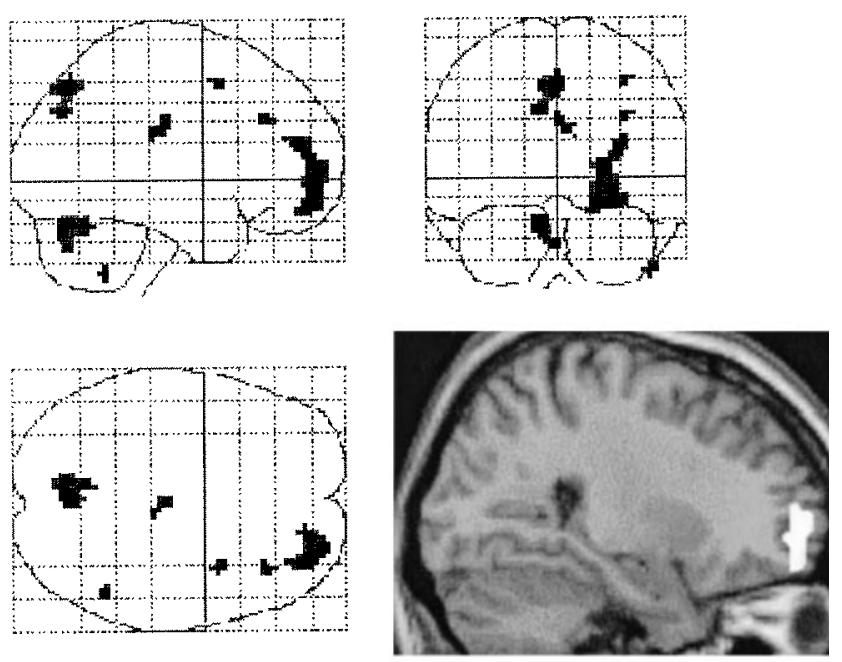

$\mathrm{X}$ coordinate $=+27$
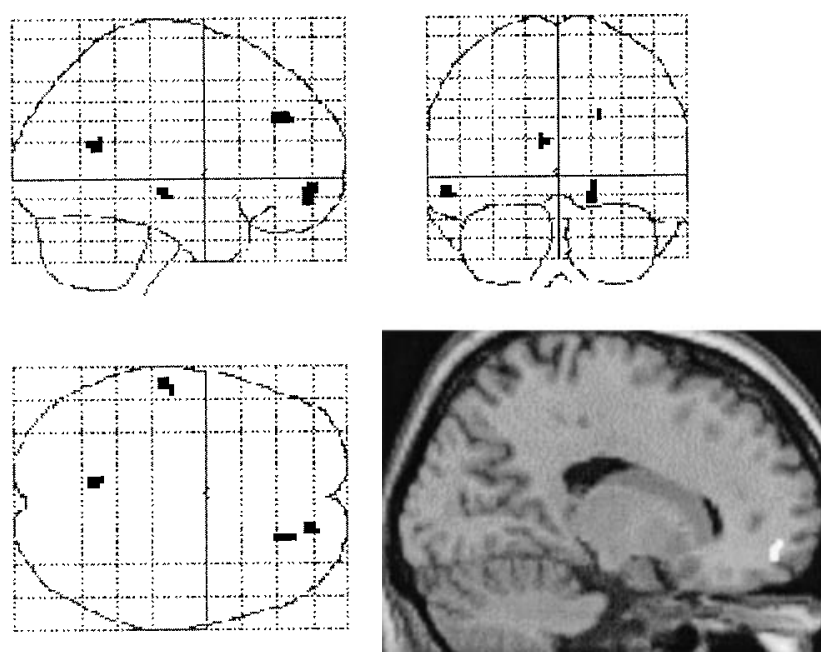

\section{$\mathrm{X}$ coordinate $=+18$}

FIG. 1. Task engagement effects (zero target - baseline) shown as maximum intensity projections (thresholded at $P<0.001$ and, for purposes of illustration, clusters of 7 or more contiguous voxels) and superimposed onto sagittal sections of a standard magnetic resonance image (top, stem cued recall; bottom, fragment cued recall). fragments and for fragments versus stems (see Table 3). Two adjacent regions of right anterior PF C exhibited a greater engagement response during stem cued recall than during fragment cued recall; one of these regions overlapped with the region of BA 10 that exhibited engagement effects in both tasks, while the other region was slightly more posterior and dorsal, though still located within BA 10. In contrast, no region of the PFC exhibited a greater engagement effect for fragments than for stems. Figure 2 illustrates the pattern of enhanced right anterior PFC activation that is associated with stem cued recall.

Data from the right anterior voxel $(x, y, z=33,51,18)$ at which the task by engagement interaction was most significant are illustrated in Fig. 3, in which it can be seen that an important determinant of this interaction appears to be the difference in activity between the two baseline tasks. Consistent with this impression, the contrast between the two baseline conditions revealed greater activity for the fragment condition, with a peak $(x, y, z=33,54,27 ; z=3.61)$ that was very close to the voxel whose activity is illustrated in Fig. 3.

As can be seen from Table 3 and Fig. 4, a greater engagement response for fragments relative to stems was observed within theright dorsal anterior hippocampal formation. This region was not identified in the pairwise comparisons described in Table 2. One interpretation of this finding is that the right hippocampal formation was deactivated during stem cued recall but not during fragment cued recall, relative to their respective baseline conditions. This possibility was tested by additional pairwise contrasts to identify regions that were more active during the baseline relative to the zero density conditions. The right hippocampal formation was more active during the baseline compared to the zero density condition for stems $(x, y, z=27,-15$, $-12 ; Z=4.24$ ) but not for fragments, supporting our interpretation. Data from the above voxel are illustrated in Fig. 5.

Activations associated with retrieval success on each task were identified by pairwise contrasts between the high and the zero target density conditions. As in the previous study of Rugg et al. (1998), these pairwise contrasts were masked by an orthogonal contrast between all of the memory conditions (high and zero target) versus the two baseline conditions. The mask was employed to increase the sensitivity of the test for memory-related increases in activity, while at the same time effecting a degree of control over type I error. The masking contrast threshold was $P<0.01(Z=2.31)$. Voxels within the mask which also exceeded a $P<0.01$ threshold in the contrasts testing for density effects were considered sensitive to target density. Note that although the $Z$ values reported in Tables 4 and 5 are given for the contrasts testing for density effects, the conjoint probability of a voxel exceeding the signifi- 
TABLE 3

$x, y$, and z Coordinates, Z Values, Brain Regions, and Approximate Brodmann Areas (BAs) of the Peak Increases in rCBF for the Interactions between the Task Engagement Effects (Zero Target Minus Baseline)

\begin{tabular}{clclll}
\hline Contrast & Coordinates & Voxels & Z & \multicolumn{1}{c}{ Region } & BA \\
\hline Stems - Fragments & $33,51,18$ & 24 & 3.92 & Right anterior prefrontal & 10 \\
& $27,67,3$ & 8 & 3.38 & Right anterior prefrontal & 10 \\
& $48,-54,-48$ & 12 & 3.76 & Left cerebellum & Left cerebellum \\
& $-21,-75,-45$ & 8 & 3.48 & Left middletemporal gyrus & $21 / 37$ \\
& $-51,-45,6$ & 13 & 3.74 & Right hippocampal formation & 40 \\
\hline
\end{tabular}

cance level for the orthogonal masking and density contrasts is approximately the multiple of the two individual thresholds, i.e., $\mathrm{P}<0.0001$.

As can be seen from Table 4, retrieval success in fragment cued recall, but not stem cued recall, was associated with activation of the right dorsolateral PFC in BA 8. The same region also exhibited a greater response to retrieval success when cued by fragments relative to stems, as revealed by the task $\times$ success (high - zero target) interactions described in Table 5 and illustrated for the right dorsolateral PFC in Fig. 6.

\section{DISCUSSION}

The probabilities of successfully completing the retrieval cues with target items, and correctly judging those items as having been presented at study, were equivalent in both tasks. Rugg et al. (1998) reported similar levels of recall in the zero target condition of their word stem cued recall task. Rugg et al. did not, however, find that the probability of successful retrieval was increased in the high relative to the zero target condition, as was the case in the present experiment. Participants in the present study may thus have detected the manipulation of target density and adjusted their performance accordingly, for example, by making less effort to retrieve in the zero target than in the high target conditions. While such an interpretation cannot be ruled out, it is inconsistent with the participants' postexperiment reports that they failed to notice the density manipulation. In any case, the factors that led to the disparity in performance between the high and the zero target conditions appear to have affected both tasks equally and are unlikely to have contributed to the task-specific patterns of activation that are the focus of the present experiment.

The rationale for employing the two classes of retrieval cue was that it would be more difficult to generate completions for the fragments than for the stems, the intention being to constrain the iterative evaluation of candidate target items during fragment cued recall relative to stem cued recall. Inspection of the don't know rates shown in Table 1 indicates that our intention was successful. Participants were unable
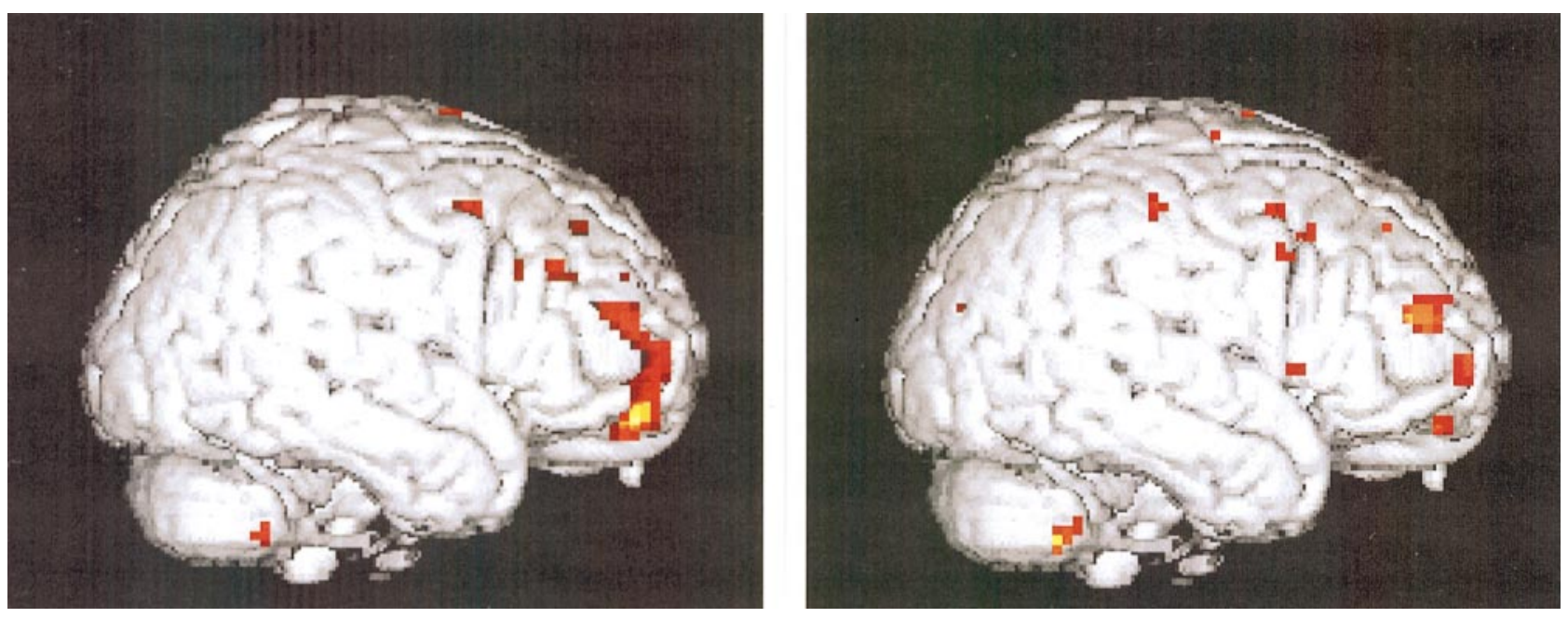

FIG. 2. Statistical parametric maps (threshold $\mathrm{P}<0.001$ ) rendered onto a standard cortical surface to illustrate right anterior $\mathrm{PFC}$ activation associated with stem cued recall. Left: Engagement effects for the stem cued recall task (zero target - baseline). Right: Engagement effects that were greater for stem than for fragment cued recall [stem (zerotarget - baseline) - fragment (zerotarget - baseline)]. 


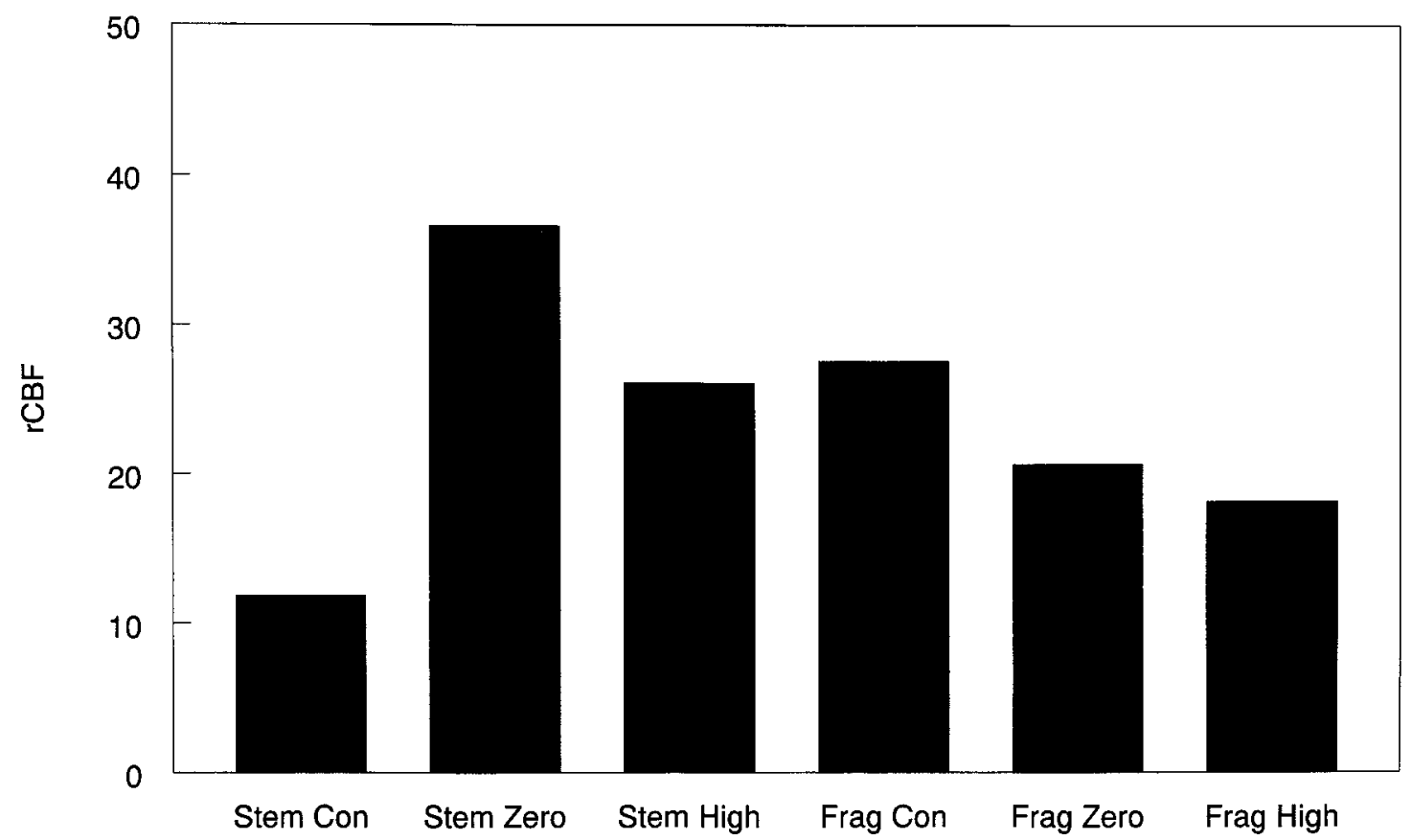

FIG. 3. Mean rCBF values (in arbitrary units adjusted for global flow) in each experimental condition from a voxel in right anterior PFC $(x, y, z=33,51,18)$.

to provide any completion for the fragment cues in approximately half of the trials, substantially more often than was the case for the stems. For fragments, but not for stems, there was a difference in the don't know rates according to target density. In the high

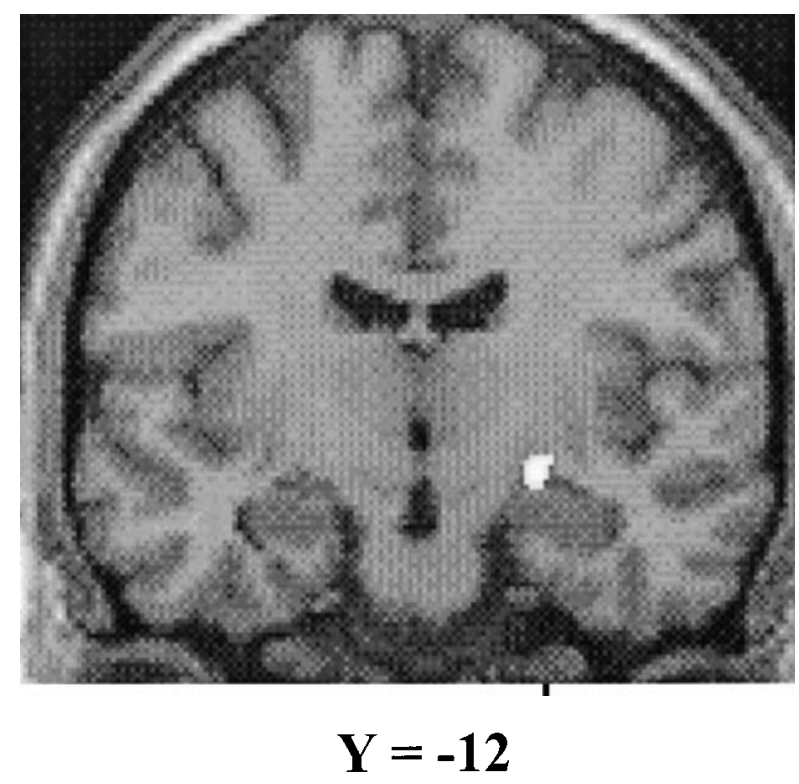

FIG. 4. Statistical parametric map (threshold $P<0.001$ ) superimposed onto a coronal section of a standard magnetic resonance brain image to illustrate relatively greater activity within the right hippocampal formation during fragment cued recall compared to stem cued recall. target fragment condition, the don't know rate was lower relative to the zero density and the baseline conditions, which did not differ from one another in this respect. This effect presumably reflects the beneficial influence on completion rates of memory for the target items. The failure to find an analogous effect in the word stem task was most likely a consequence of the near-floor don't know rates in that task.

In both tasks, right anterior PF C activity was greater in the zero target conditions compared to their respective baselines (see Figs. 1 and 2). As predicted by the monitoring hypothesis, this "task engagement effect" was greater for stems than for fragments. A further prediction of the monitoring hypothesis was that right anterior PFC activity would be greater in the high compared to the zero target condition during fragment cued recall. Contrary to this prediction, however, we found that right anterior PFC activity did not differ reliably between the high and the zero target conditions in either task. In fragment cued recall, however, a region of dorsolateral prefrontal cortex (BA 8) was more active in the high relative to the zero target condition (see Fig. 6).

The monitoring hypothesis combines el ements of two previous functional interpretations of the role of the right PFC in episodic memory, the retrieval "success" (e.g., Rugg et al., 1996) and retrieval "effort" (e.g., Schacter et al., 1996) accounts. The retrieval success account linked right anterior PFC activity to cognitive operations that are engaged when details of a past 


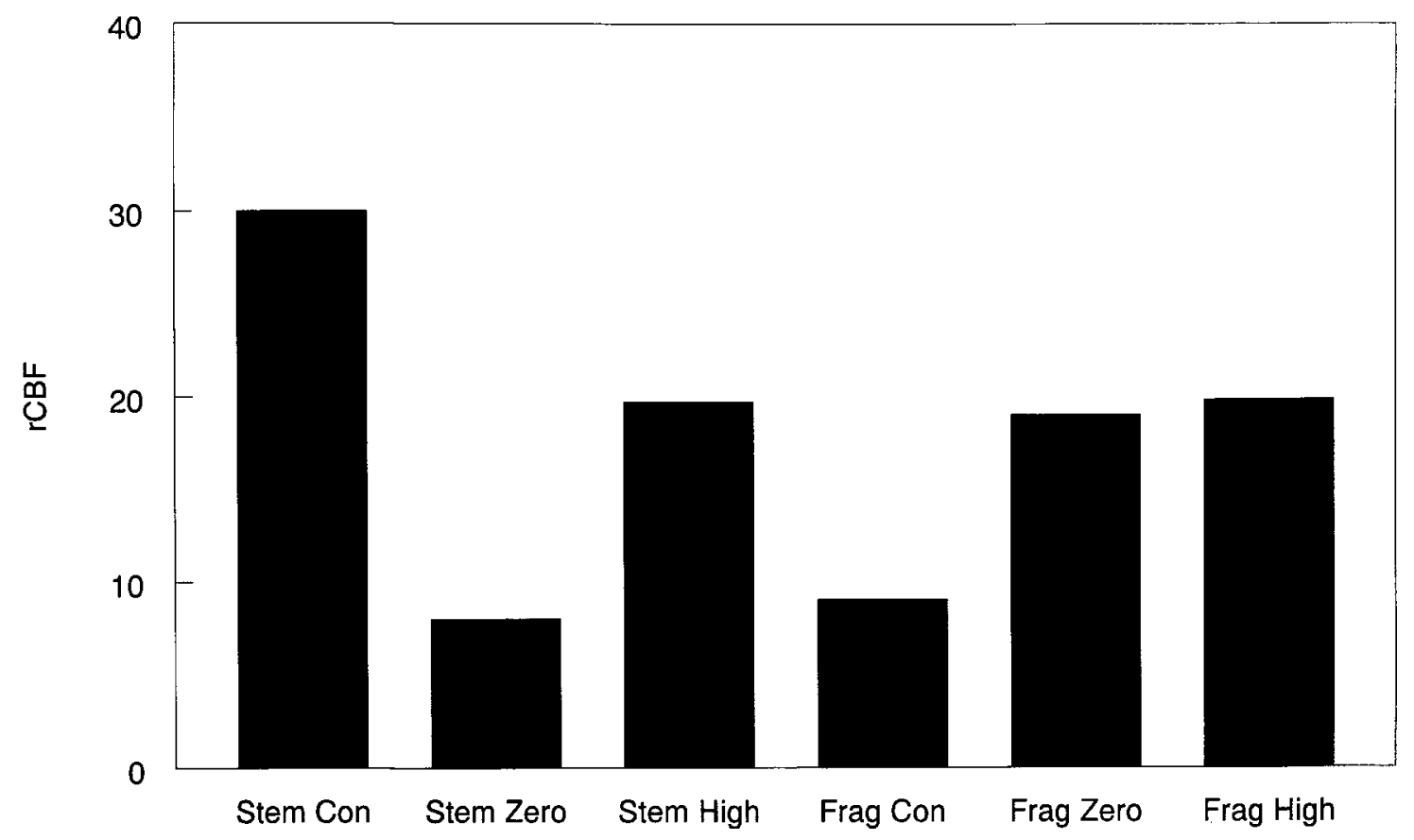

FIG. 5. Mean rCBF values (in arbitrary units adjusted for global flow) in each experimental condition from a voxel in the right hippocampal formation $(x, y, z=27,-15,-12)$.

event are recollected. A critical failing of the account was its inability to explain increased right PFC activity during conditions in which veridical retrieval of a study episode was by definition impossible. That is, in the zero target conditions of the present experiment and the zero target stem cued recall condition in the study of Rugg et al. (1998).

As al ready outlined, one aim of the present study was to provide a further test of the proposal (Rugg et al., 1998) that activation of the right PFC during the processing of zero target test lists reflects monitoring operations following attempted retrieval. Thus, we predicted that word stems, which allow multiple re trieval attempts to be initiated with a single retrieval cue, would give rise to greater activation in zero target lists than would word fragments, for which this iterative strategy is not available. The monitoring hypothesis stands in contrast to the effort (Schacter et al.,
1996) and "mode" (e.g., Duzel et al., 1999; Nyberg et al., 1995; Wheel er et al., 1997) hypotheses al so advanced to account for activation of the anterior right PFC during episodic retrieval. According to the former account, right PFC activation is proportional to the processing resources allocated to an episodic retrieval search (but see Buckner et al., 1998; Wagner et al., 1998; Rugg et al., 1998; for data incompatible with this account). According to the mode hypothesis, right PFC activation reflects the adoption of a tonically maintained state, "episodic retrieval mode," without which stimulus events cannot operate as episodic retrieval cues (Tulving, 1983)

At first glance, the pattern of task engagement effects in the right anterior PFC observed in the present experiment is difficult to reconcile with either the effort or the mode accounts. As predicted by the monitoring hypothesis, right PFC activity was more sensitive to

\section{TABLE 4}

$x, y$, and z Coordinates, Z Values, Brain Regions, and Approximate Brodmann Areas (BAs) of the Peak Increases in rCBF for the Masked Pairwise Contrasts between the High and the Zero Target Conditions for Each Task

\begin{tabular}{clcclc}
\hline \multicolumn{1}{c}{ Contrast } & Coordinates & Voxels & Z & Region & BA \\
\hline Stem cued recall & $-45,48,3$ & 5 & 2.72 & Left anterior prefrontal & $10 / 46$ \\
& $-6,-57,15$ & 7 & 2.70 & Left posterior cingulate & 31 \\
Fragment cued recall & $33,27,39$ & 8 & 2.68 & Right middlefrontal gyrus & 8 \\
& $-39,-63,42$ & 12 & 3.86 & Left lateral parietal & $19 / 40$ \\
& $-18,-54,39$ & 11 & 3.20 & Left medial parietal & 7 \\
& $-69,-36,-6$ & 9 & 3.26 & Left middle temporal gyrus & 21 \\
\hline
\end{tabular}


TABLE 5

$x, y$, and z Coordinates, Z Values, Brain Regions, and Approximate Brodmann Areas (BAs) of the Peak Increases in rCBF for the Masked Interaction between the Retrieval Success Effects (High Minus Zero Target) for Each Task

\begin{tabular}{clcclc}
\hline Contrast & Coordinates & Voxels & Z & Region & BA \\
\hline Stems - Fragments & $-6,-57,15$ & 7 & 3.36 & Left posterior cingulate & $23 / 31$ \\
& $-45,48,3$ & 7 & 3.76 & Left anterior prefrontal & $10 / 46$ \\
Fragments - Stems & $33,30,39$ & 18 & 2.94 & Right middle frontal gyrus & 8 \\
& $24,-15,66$ & 6 & 2.84 & Right postcentral gyrus & 4 \\
& $3,-69,54$ & 7 & 2.62 & Right lateral parietal sulcus/precuneus & 7 \\
\hline
\end{tabular}

task engagement in the stem than in the fragment recall task. Yet there is no obvious reason why the former task should be regarded as the more effortful (indeed, subjectively, the reverse was the case). Nor is it clear how the mode hypothesis can accommodate the finding of differences in right anterior PFC activity between two such similar episodic retrieval tasks. A complication in the interpretation of these results arises, however, because right prefrontal activity differed between the two baseline tasks (see Fig. 3).

The finding of greater activity in the fragment baseline task raises the possibility that the smaller engagement effect for fragment cued recall has as much as or more to do with the demands of the baseline task than the zero target memory condition. For example, it may be that the difficulty in finding suitable completions in the fragment baseline task led subjects to adopt the strategy of searching episodic memory for recently encountered candidate words that matched the frag-

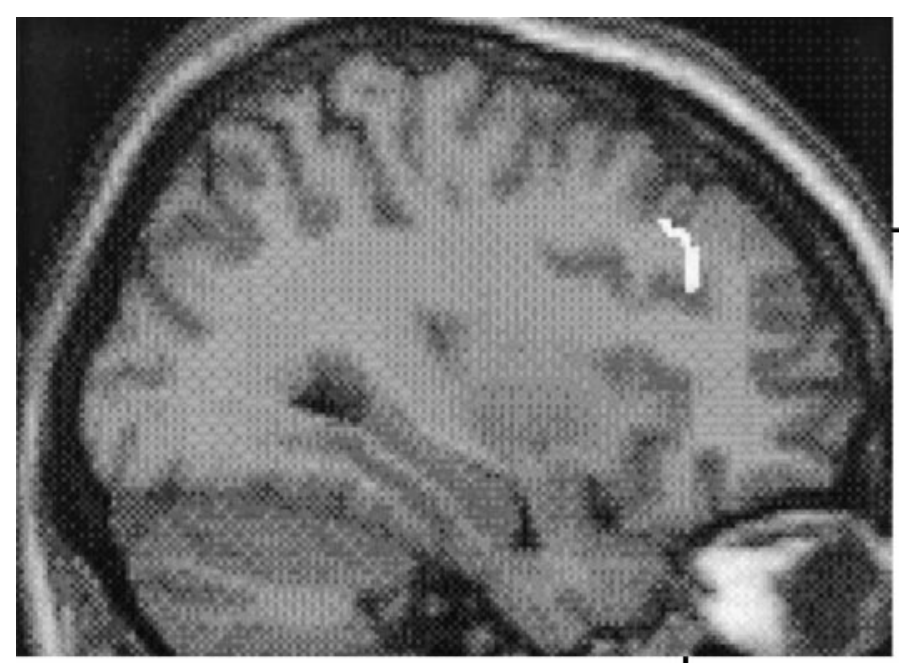

$$
X=+33
$$

FIG. 6. Statistical parametric map (threshold approximately $\mathrm{P}<0.0001$ ) superimposed onto a sagittal slice of a standard magnetic resonance brain image illustrating the masked retrieval success (high target - zero target) interaction effect for fragments versus stems within the right prefrontal cortex. ments. If so, it would of course be inappropriate to treat fragment completion as a nonepisodic basel ine task.

The reasons for the differences in right PFC activity between the two baseline tasks cannot be determined on the basis of the present data. The presence of these differences leaves open the possibility that the differential effects of task engagement observed in the present study do not reflect differences in the monitoring demands imposed by stem and fragment retrieval cues. We would argue nonetheless that the monitoring hypothesis provides the most parsimonious account of the task sensitivity of the engagement effects observed both in the present study and in our previous report (Rugg et al., 1998), when the same problem of interpretation does not arise.

According to the monitoring hypothesis, the successful retrieval of information belonging to a past event should also be associated with monitoring operations. In the present study, however, we failed to find evidence for increased right anterior PFC activity associated with higher levels of retrieval success on either the stem or the fragment cued recall tasks. Instead, we found that during fragment cued recall a dorsolateral region of right PFC (BA 8) was more active in the high relative to the zero target condition.

Why was the right anterior PFC insensitive to the manipulation of retrieval success during fragment cued recall? One possibility is that the level of successful recall during the high target condition (54.2\%) was too low to tax monitoring operations to a greater extent than was the case in the zero target condition. Another possibility is that multiple monitoring and evaluative operations exist (e.g., Koriat and Goldsmith, 1996), with different operations engaged during successful and unsuccessful cued recall. Differences may arise, for example, according to the kind of information that is subject to monitoring and evaluation when retrieval is successful compared to when it is not. Thus, the present findings suggest, as do the findings of other neuroimaging (e.g., Fletcher et al., 1998; Henson et al., 1999a) and neuropsychological (see Shallice, 1988) studies, that it may be possibleto fractionatefunctionally the contribution made by different regions of the PFC during episodic memory retrieval. Fletcher et al. (1998) and 
Henson et al. (1999a) have, for example, proposed that retrieval monitoring may involve BA 46/9 in the right dorsolateral PFC, while the ventrolateral right PFC (BA 45) may be involved in specifying retrieval cues. But the present experiment shows that differences in retrieval monitoring are also associated with variation in right anterior PFC activity. A principled explanation of these across-experiment differences in the loci of activations within the right PFC is an important goal for future research.

\section{H ippocampal Formation}

The right hippocampal formation was relatively less active during stem cued recall compared to its baseline condition, a pattern that was not present for fragment cued recall (see Fig. 3). Previous studies of stem completion and cued recall have also demonstrated right hippocampal activation (Squire et al., 1992; Schacter et al., 1996; but see Buckner et al., 1995). H owever, Squire et al. (1992) reported results exactly contrary to our own, in that they found increased right hippocampal activity during stem cued recall relative to a stem completion baseline condition. The findings of Schacter et al . (1996) were also in essence contrary to our own, in that these authors reported increased right hippocampal activation for high relative to low levels of retrieval success during stem cued recall. In addition, Rugg et al . (1998), in their previous study of stem cued recall and recognition memory, did not report differential activity within the hippocampal formation in either task. These previous studies do not, therefore, provide any clue as to the functional significance of the present finding.

Indeed, the exact role of the hippocampal region during memory retrieval is the subject of vigorous debate (Lepage et al., 1998; Tulving et al., 1999). One proposal (e.g., Dolan and Fletcher, 1997; Tulving et al., 1994) is that the hippocampal region acts as a "novelty" detector. However, it is not clear how this interpretation would relate to the present finding. For example, consider the zero target stem condition. In this case, why should word stems that do not belong to any studied items engender greater novelty when they are presented in a completion task than in a cued recall task? F urthermore, why should fragments presented in the context of completion and cued recall tasks not differ in terms of their novelty if stems do so? The present finding thus adds to the current debate concerning the function of the hippocampal region during retrieval, but does nothing to help resolve it.

\section{Left PFC}

In a restricted region of the left anterior PFC, activity was increased during stem but not fragment cued recall in the zero relative to the baseline condition (see Table 2) and in the high target relative to the zero target condition (see Tables 4 and 5). These findings suggest that the left anterior PFC may be particularly important for recall cued by stems compared to fragments. These findings do not, however, exactly replicate those of Rugg et al. (1998) for stem cued recall. Rugg et al. found that activity within the left anterior PFC was lower in the high target relative to the zero target condition.

Modulations of left anterior PFC activity have been observed in a number of previous imaging studies of memory retrieval (for reviews see Buckner and Petersen, 1996; Fletcher et al., 1997), but a detailed functional account of the role of the left anterior PFC during retrieval has yet to emerge. Henson et al. (1999b) have recently suggested that the region may be involved in maintaining retrieved verbal information in working memory (for a related view see $\mathrm{N}$ ol de et al., 1998). The present findings are not inconsistent with such proposals, and in addition they suggest that the natures of the retrieved information that is accessed with stem and fragment cues may differ. Specifically, more verbal information may be retrieved, or be the focus of greater attention, during stem compared to fragment cued recall. It is possible that the failure of Rugg et al. (1998) to observe increased left anterior PFC activity when contrasting the high with the zero target condition reflected the slightly lower level of successful retrieval in the high target condition in that study compared to the present one.

\section{Parietal C ortex}

As in our previous study (Rugg et al., 1998), increases in the activity of medial and lateral parietal regions were associated with task engagement and retrieval success during stem cued recall. The present findings indicate that the same regions are also sensitive to these two factors during fragment cued recall. N otably, medial parietal activity was higher in the high target relative to the zero target condition for fragment cued recall, but not for stem cued recall (see Tables 4 and 5). Some authors have suggested that medial parietal cortex may be involved in the retrieval of visual form or imagery information (Grasby et al., 1993; Fletcher et al., 1995b, 1996; but see Krause et al., 1999). It is possible therefore that there is a difference in the nature of the information that is successfully retrieved with stem and fragment cues, with visual imagery playing a relatively greater role during recall cued by fragments than by stems.

Rugg et al. (1998) reported greater medial parietal activity when contrasting the high compared to the zero target condition during stem cued recall, but we failed to replicate this finding in the present experiment. It seems unlikely that this failure reflects differences in the level of retrieval success across the two experiments, because performance in the study of Rugg et al. was if anything slightly lower than was the case in the present experiment. This disparity across the two studies suggests that the contribution of medial pari- 
etal cortex during retrieval may not simply reflect retrieval success, but may be modulated by other factors. One possibility, al beit speculative, is that switching between the fragment and the stem cued recall tasks from one scan to the next may have encouraged the participants in the present study to adopt taskspecific retrieval strategies, focusing attention upon different aspects of retrieved information in each task. It is not unreasonable to suppose, then, that preferential retrieval of verbal information may have occurred during stem cued recall, while greater reliance may have been made upon the retrieval of visual imagery information during fragment cued recall.

\section{ACKNOWLEDGMENT}

This research and the authors were supported by the Wellcome Trust.

\section{REFERENCES}

Buckner, R. L., and Koutstaal, W. 1998. Functional neuroimaging studies of encoding, priming, and explicit memory retrieval. Proc. Natl. Acad. Sci. USA 95:891-898.

Buckner, R. L., Koutstaal, W., Schacter, D. L., Wagner, A. D., and Rosen, B. R. 1998. Functional-anatomic study of episodic memory using fMRI. I. Retrieval effort versus retrieval success. Neurol mage 7:151-162.

Buckner, R. L., and Petersen, S. E. 1996. What does neuroimaging tell us about the role of prefrontal cortex in memory retrieval? Semin. Neurosci. 8:47-55.

Buckner, R. L., Petersen, S. E., Ojemann, J . G., Miezin, F. M., Squire, L. R., and Raichle, M. E. 1995. Functional anatomical studies of explicit and implicit memory retrieval tasks. J . Neurosci. 15:12-29.

Dolan, R. J ., and Fletcher, P. C. 1997. Dissociating prefrontal and hippocampal function in episodic memory encoding. Nature 388: 582-585.

Duzel, E., Cabeza, R., Picton, T. W., Yonelinas, A. P., Scheich, H., Heinze, H. J ., and Tulving, E. 1999. Task-related and item-related brain processes of memory retrieval. Proc. Natl. Acad. Sci. USA 96:1794-1799.

Fletcher, P. C., Frith, C. D., Grasby, P. M., Shallice, T., Frackowiak, R. S. J ., and Dolan, R. J . 1995a. Brain systems for encoding and retrieval of auditory-verbal memory. An in vivo study in humans. Brain 118:401-416.

Fletcher, P. C., Frith, C. D., Baker, S. C., Shallice, T., Frackowiak, R. S. J., and Dolan, R. J. 1995b. The mind's eye-Precuneus activation in memory-related imagery. Neurol mage 2:195-200.

Fletcher, P. C., Frith, C. D., and Rugg, M. D. 1997. Functional neuroanatomy of episodic memory. Trends Neurosci. 20:213-218.

Fletcher, P. C., Shallice, T., Frith, C. D., Frackowiak, R. S. J ., and Dolan, R. J. 1988. The functional roles of prefrontal cortex in episodic memory. II. Retrieval. Brain 121:1249-1256.

Fletcher, P. C., Shallice, T., Frith, C. D., Frackowiak, R. S. J ., and Dolan, R. J . 1996. Brain activity during memory retrieval: The influence of imagery and semantic cueing. Brain 119:1587-1596.

Grasby, P. M., Frith, C. D., Friston, K.J ., Simpson, J ., Fletcher, P. C., Frackowiak, R. S. J ., and Dolan, R. J . 1993. Functional mapping of brain areas implicated in auditory-memory function. Brain 117: 1271-1282.

Henson, R. N. A., Shallice, T., and Dolan, R. J . 1999a. Right prefrontal cortex and episodic memory retrieval: A functional MRI test of the monitoring hypothesis. Brain 122:1367-1381.
Henson, R. N. A., Rugg, M. D., Shallice, T., J osephs, O., and Dolan, R. J . 1999b. Recollection and familiarity in recognition memory: An event-related functional magnetic resonance imaging study. J . Neurosci. 19:3962-3972.

Keselman, H. J ., and Rogan, J . C. 1980. Repeated measures F-tests and psychophysiological research: Controlling the number of false positives. Psychophysiology 17:499-503.

Koriat, A., and Goldsmith, M. 1996. Monitoring and control processes in the strategic regulation of memory accuracy. Psychol. Rev. 103:490-517.

Krause, B. J ., Schmidt, D., Mottaghy, F. M., Taylor, J ., Halsband, U., Herzog, H., Tellmann, L., and Muller-Gartner, H.-W. 1999. Episodic retrieval activates the precuneus irrespective of the imagery content of word pair associates: A PET study. Brain 122:255-263.

Lepage, M., Habib, R., and Tulving, E. 1998. Hippocampal PET activations of memory encoding and retrieval: The HIPER model. Hippocampus 8:313-322.

Nolde, S. F., J ohnson, M. K., and Raye, C. L. 1998. The role of prefrontal cortex during tests of episodic memory. Trends Cognit. Sci. 2:399-406.

Nyberg, L., Tulving, E., Habib, R., Nilsson, L.-G., Kapur, S., Houle, S., Cabeza, R., and Mclntosh, A. R. 1995. Functional brain maps of retrieval mode and recovery of episodic information. NeuroReport 7:249-252.

Rugg, M. D., Fletcher, P. C., Frith, C. D., Frackowick, R. S. J ., and Dolan, R. J . 1996. Differential activation of the prefrontal cortex in successful and unsuccessful memory retrieval. Brain 119:20732083.

Rugg, M. D., Fletcher, P. C., Allan, K., Frith, C. D., Frackowick, R. S. J., and Dolan, R. J. 1998. Neural correlates of memory retrieval during recognition memory and cued recall. Neurol mage 8:262-273.

Schacter, D. L., Alpert, N. M., Savage, C. R., Rauch, S. L., and Albert, M. S. 1996. Conscious recollection and the human hippocampal formation: Evidence from positron emission tomography. Proc. Natl. Acad. Sci. USA 93:321-325.

Shallice, T. 1988. From Neuropsychology to Mental Structure Cambridge Univ. Press, Cambridge, UK.

Silbersweig, D. A., Stern, E., Frith, C. D., Cahill, C., Schnorr, L., Grootoonk, S., Spinks, T., Clark, J ., Frackowiak, R. S. J ., and J ones, T. 1993. Detection of 30 second cognitive activations in single subjects with positron emission tomography: A new low-dose $\mathrm{H}_{2}{ }^{15} \mathrm{O}$ regional cerebral blood flow three dimensional imaging technique. J . Cereb. Blood Flow Metab. 13:617-629.

Squire, L. R., Ojemann, J . G., Miezin, F. M., Petersen, S. E., Videen, T. O., and Raichle, M. E. 1992. Activation of the hippocampus in normal humans: A functional anatomical study of memory. Proc. Natl. Acad. Sci. USA 89:1837-1841.

Talairach, J ., and Tournoux, P. 1988. Co-planar Stereotaxic Atlas of theHuman Brain. Thieme, Stuttgart.

Tulving, E. 1983. Elements of Episodic Memory. Oxford Univ. Press, Oxford.

Tulving, E., Habib, R., Nyberg, L., Lepage, M., and Mcl ntosh, A. R. 1999. Positron emission tomography correlations in and beyond medial temporal lobes. Hippocampus 9:71-82.

Tulving, E., Markowitsch, H. J ., Kapur, S., Habib, R., and Houle, S. 1994. Novelty encoding networks in the human brain: Positron emission tomography data. NeuroReport 5:2525-2528.

Wagner, A. D., Desmond, J . E., Glover, G. H., and Gabrieli, J . D. E. 1998. Prefrontal cortex and recognition memory: Functional-MRI evidencefor context-dependent retrieval processes. Brain 121:19852002.

Wheeler, M. A., Stuss, D. T., and Tulving, E. 1997. Toward a theory of episodic memory: The frontal lobes and autonoetic consciousness. Psychol. Bull. 121:331-354. 